Old Dominion University

ODU Digital Commons

Electrical \& Computer Engineering Faculty

Publications

Electrical \& Computer Engineering

2019

\title{
The Effect of Tube Geometry on the Chiral Plasma
}

S. Jin

D. Zou

X. Lu

Mounir Laroussi

Old Dominion University, mlarouss@odu.edu

Follow this and additional works at: https://digitalcommons.odu.edu/ece_fac_pubs

Part of the Electrical and Computer Engineering Commons, and the Plasma and Beam Physics

Commons

Original Publication Citation

Jin, S., Zou, D., Lu, X., \& Laroussi, M. (2019). The effect of tube geometry on the chiral plasma. Physics of Plasmas, 26(9), 093507. doi:10.1063/1.5120288

This Article is brought to you for free and open access by the Electrical \& Computer Engineering at ODU Digital Commons. It has been accepted for inclusion in Electrical \& Computer Engineering Faculty Publications by an authorized administrator of ODU Digital Commons. For more information, please contact digitalcommons@odu.edu. 


\title{
The effect of tube geometry on the chiral plasma
}

Cite as: Phys. Plasmas 26, 093507 (2019); doi: 10.1063/1.5120288

Submitted: 17 July 2019 . Accepted: 30 August 2019 .

Published Online: 12 September 2019
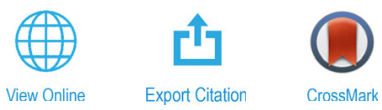

S. Jin, ${ }^{1}$ D. Zou, ${ }^{2}$ X. Lu, ${ }^{1, a)}$ (D) and M. Laroussi ${ }^{3}$

\begin{abstract}
AFFILIATIONS
${ }^{7}$ State Key Laboratory of Advanced Electromagnetic Engineering and Technology, School of Electrical and Electronic Engineering, Huazhong University of Science and Technology, Wuhan, HuBei 430074, People's Republic of China

${ }^{2}$ School of Electrical and Automation Engineering, East China Jiaotong University, Nanchang, Jiangxi 330013, People's Republic of China

${ }^{3}$ Electrical and Computer Engineering Department, Old Dominion University, Norfolk, Virginia 23529, USA
\end{abstract}

a) Author to whom correspondence should be addressed: Iuxinpei@hotmail.com

\begin{abstract}
A chiral plasma plume has recently been reported inside a circular quartz tube without the use of an external magnetic field. It is believed that the quartz tube plays an important role in the formation of the chiral plasma plume. In this paper, to better understand how this interesting structure is generated, the effect of the tube geometry on the chiral plasma is investigated. First, the effect of the thickness of the tube wall on the chiral plasma is investigated. It is interesting to find that a too thin or too thick tube wall is not favorable for generating the chiral plasma. The chiral plasma plume can be obtained only at the tube wall thickness of $2 \mathrm{~mm}$. Second, the effect of the diameter of the tube on the chiral plasma is investigated. It is found that, when a tube with an inner diameter of $2 \mathrm{~mm}$ is connected with a tube with an inner diameter of $4 \mathrm{~mm}$ and the high voltage electrode is placed in the tube with an inner diameter of $2 \mathrm{~mm}$, a diffuse plasma is generated in the tube with an inner diameter of $2 \mathrm{~mm}$, it propagates into the tube with an inner diameter of $4 \mathrm{~mm}$, and a chiral plasma can be obtained at a distance of about $2 \mathrm{~cm}$ away from the joint of the two tubes. Third, to further understand how the tube diameter affects the appearance of the chiral plasma, horn shaped tubes with different opening angles are tested. It is found that, when the opening angle is $5^{\circ}$, the chiral plasma formation in the straight part of the tube can maintain the chiral shape and keep propagating in the horn shaped tube but with a much longer pitch. Fourth, to disrupt the axial symmetry, square quartz tubes are used. No chiral plasma can be obtained in the square quartz tubes under all experimental conditions. In addition, when a circular quartz tube is connected with a square tube, the chiral plasma formation in the circular quartz tube changes to a straight plasma plume in the square tube. Finally, the high voltage electrode is placed away from the center of the circular tube to disrupt its symmetry, and it is found that the chiral plasma plume can always be seen even if the high voltage is placed next to the inner wall of the tube.
\end{abstract}

Published under license by AIP Publishing. https://doi.org/10.1063/1.5120288

\section{INTRODUCTION}

Atmospheric pressure plasma jets propagate along a predetermined path defined by the flow of noble gases. ${ }^{1-8}$ They normally appear as straight plasma plumes and are widely used for various applications such as plasma medicine. ${ }^{9-16}$ High speed photographs show that the plasma is actually like a "bullet" with a propagation velocity of $10^{4}$ to $10^{5} \mathrm{~m} / \mathrm{s}^{17-23}$ Under some specific conditions, the plasma plume could also exhibit different shapes such as a featherlike shape. ${ }^{24}$

Chiral plasma mostly appears in devices such as tokamaks and stellarators where the external magnetic field is applied. However, as reported in our previous work, a chiral shaped plasma driven by pulsed DC voltages without the external magnetic field at subatmospheric-pressure nitrogen gas has been observed recently. ${ }^{25}$ In addition, similar stable helix plasmas are also found in other plasma devices. $^{26,27}$ However, the reason for the appearance of the helix plasma is still not well understood.

In general, when a plasma is generated in a dielectric tube, it is believed that the dielectric tube will affect the plasma because of the charge accumulation on the inner surface of the quartz tube. ${ }^{28-30}$ Investigations on the dynamic behavior of the plasmas generated in the tubes showed that most of them propagate in a straight line. This is totally different from what is reported in Ref. 25 where a stable helix plasma plume is generated inside a quartz tube.

This chiral shape only appears under limited experimental conditions. When the gas pressure or the electrical parameters (amplitude, frequency, and pulse width of the applied voltage) are changed slightly, the chiral plasma disappears. Further studies show that, when a stainless steel helical coil is placed on the outside of the dielectric tube, the 
chiral plasma appears in much wider parameter ranges than the case without the helical coil. ${ }^{31,32}$ It is suspected that the helical coil reduces the impedance of the discharge channel and thus results in the appearance of the chiral plasma. However, it is still not clearly understood what contributes to the initiation of the chiral plasma when no helical coil is used.

As reported previously, it is believed that the dielectric tube plays an important role in the discharge behavior. However, for all the experiments reported, only circular tube has been used. Since the dielectric tube plays an important role in the appearance of the chiral plasma, in this paper, the dimensions of the tube, the shape of the tube, and the position of the high voltage electrode are adjusted to see how they affect the appearance of the chiral plasma. It is believed that all the phenomena reported in this paper will be helpful for a better understanding of the mechanism of the chiral plasma.

\section{EXPERIMENTAL SETUP}

The schematic of the experimental setup is shown in Fig. 1(a). The vacuum chamber is connected to the gas inlet and vacuum pump. Nitrogen is used as the working gas. Various discharge tubes as shown in Figs. 1(b)-1(f) are placed in the vacuum chamber. The plasma plume is generated in all the quartz tubes. A needle electrode is inserted into one end of the quartz tube. The other end of the quartz tube is open. Five different groups of quartz tubes are used in the experiment. Figure 1(b) shows quartz tubes with different wall thicknesses. Figure 1(c) shows two quartz tubes with different diameters connected together. Figure 1 (d) shows quartz tubes with a horn shape of different angles. Figure $1(\mathrm{e})$ is a square shaped quartz tube. Figure $1(f)$ is a tube where the left part of the tube has a circular shape, while the right part has a square shape. The total length of all the quartz tubes is $10 \mathrm{~cm}$.

A pulsed DC power supply with an adjustable amplitude of $0-10 \mathrm{kV}$, a repetition frequency of $0-10 \mathrm{kHz}$, and a pulse width from 200 ns to DC is used in this experiment. A Tektronix HV probe P6015 is used to measure the applied voltage. The gas pressure in the vacuum pump is measured using an Inficon vacuum gauge PCG550. The plasma photographs are captured using a Canon D7000 camera with an exposure time of $0.1 \mathrm{~s}$.

\section{EXPERIMENTAL RESULTS}

\section{A. The effect of gas pressure and electrical parameters on the chiral plasma}

In this experiment, a quartz tube with inner and outer diameters of 4 and $8 \mathrm{~mm}$, respectively, is used. The gas pressure in the vacuum chamber and the electrical parameters are adjusted. The corresponding photographs of the plasmas are captured.

\section{The effect of gas pressure on the chiral plasma}

The gas pressure in the vacuum chamber is adjusted from $3 \mathrm{kPa}$ to $11 \mathrm{kPa}$, and the corresponding photographs of the plasmas are captured. As shown in Fig. 2, when the gas pressure is $3 \mathrm{kPa}$, a diffuse plasma can be observed. With the increase in gas pressure to $5 \mathrm{kPa}$, a blurry chiral plasma can be observed. Upon a further increase in the gas pressure to $7 \mathrm{kPa}$, a clear and long chiral plasma appears. However, upon increasing the gas pressure to $9 \mathrm{kPa}$, a clear chiral plasma can still be observed, but it becomes shorter. When the gas pressure is increased to $11 \mathrm{kPa}$, the chiral plasma becomes blurry again, and it is much shorter. (a)

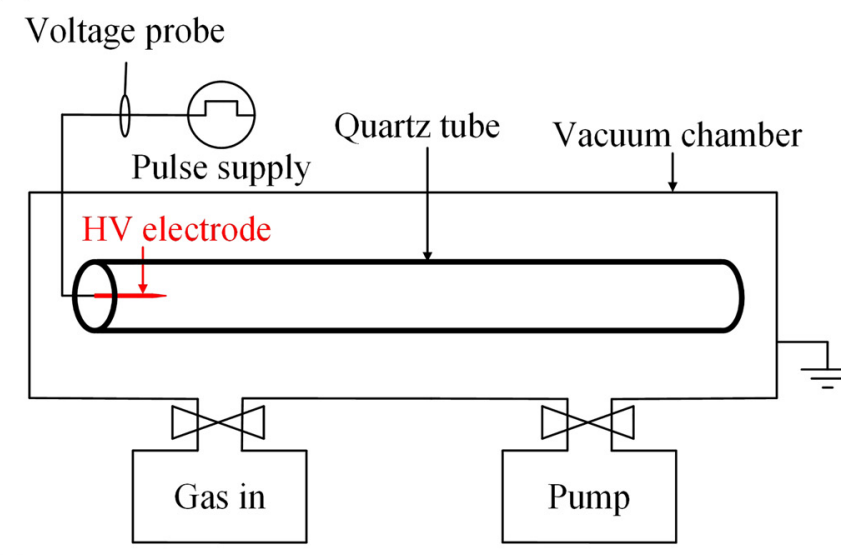

(b)

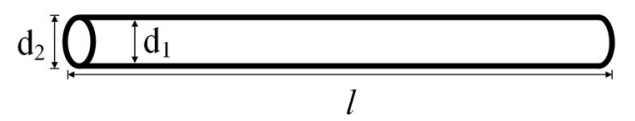

(c)

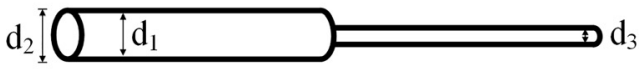

(d)

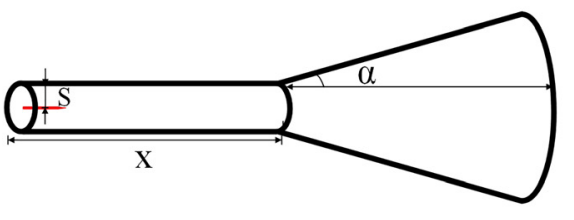

(e)

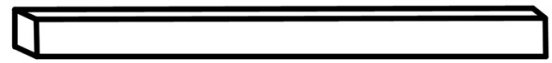

(f)

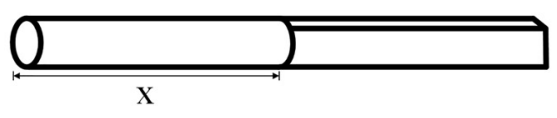

FIG. 1. (a) Schematic of the experimental setup. (b) Quartz tubes with different inner and outer diameters. (c) Two quartz tubes with different diameters connected together. (d) Quartz tube with the needle electrode place shifted from the center. Besides, the quartz tubes have a horn shape of different angles. (e) Square shaped quartz tube. ( $f$ ) Quartz tube where the left part of the tube has a circular shape, while the right part has a square shape. 


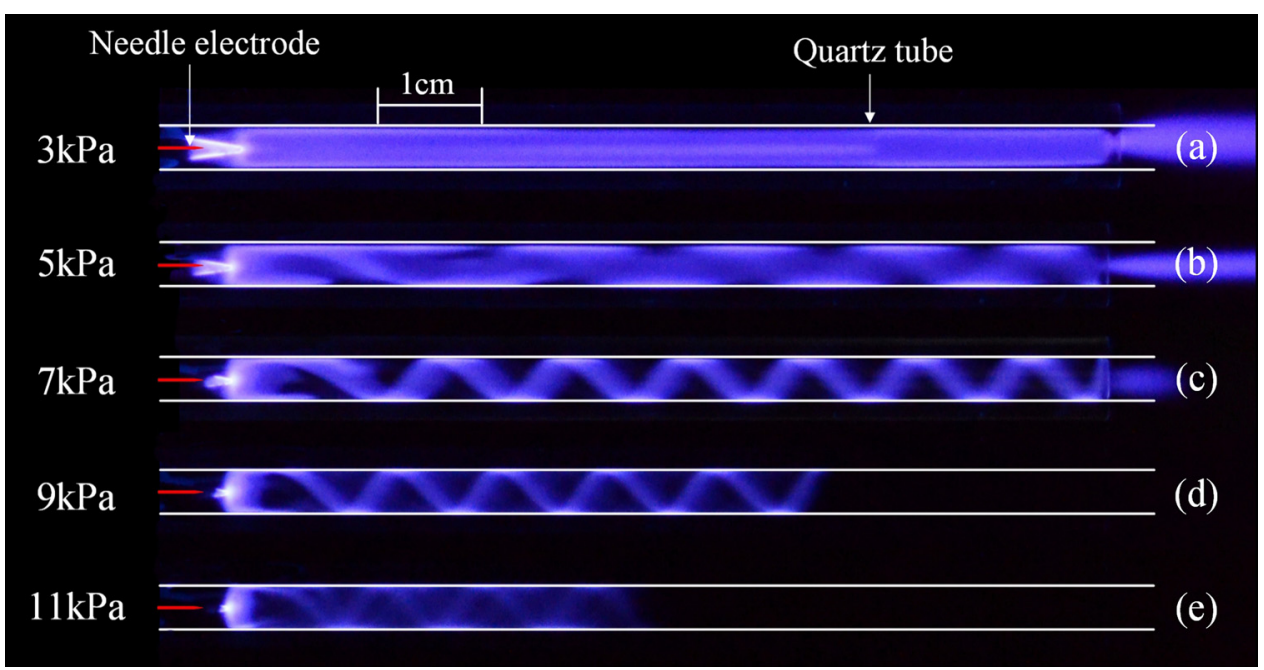

FIG. 2. Photographs of the plasma with different gas pressures. (a)-(e) correspond to the gas pressures of $3,5,7,9$, and $11 \mathrm{kPa}$, respectively. The inner and outer diameters of the tube are 4 and $8 \mathrm{~mm}$, and the total length of the quartz tube is $10 \mathrm{~cm}$. Working gas: $\mathrm{N}_{2}$; amplitude of voltage: $4.5 \mathrm{kV}$, pulse frequency: $2 \mathrm{kHz}$, and pulse width: $1 \mu \mathrm{s}$.

\section{The effect of applied voltage amplitude on the chiral plasma}

The applied voltage is adjusted from $4 \mathrm{kV}$ to $5.5 \mathrm{kV}$, and the corresponding photographs of the plasmas are captured as shown in Fig. 3. When the applied voltage is $4 \mathrm{kV}$, the chiral plasma can be observed. When it is increased to $4.5 \mathrm{kV}$, the length of the chiral becomes longer, and the chiral shape can still be clearly seen. However, when the voltage is further increased to $5 \mathrm{kV}$, the plasma becomes blurry and the chiral shape becomes unclear. Upon further increasing the applied voltage to $5.5 \mathrm{kV}$, the plasma appears more or less diffused.

\section{The effect of the pulse width of the applied voltage on the chiral plasma}

The pulse width of the applied voltage is adjusted from $0.5 \mu$ s to $10 \mu \mathrm{s}$, and the corresponding photographs of the plasma are captured as shown in Fig. 4. When the pulse width is set as $0.5 \mu \mathrm{s}$, the chiral plasma can be observed. Increasing the pulse width to $1 \mu$ s results in a longer chiral plasma plume. But upon further increasing the pulse width to $2 \mu \mathrm{s}$, the plasma appears dimmer and the chiral shape becomes unclear. Increasing the pulse width to 5 or $10 \mu$ s results in a significant reduction in the length of the plasma plume.

\section{The effect of the pulse frequency of the applied voltage on the chiral plasma}

Figure 5 shows the photograph of the plasma for pulse frequencies of $1,3,5$, and $7 \mathrm{kHz}$, respectively. As shown in Figs. 5(a)-5(c), with the increase in the pulse frequency, the chiral plasma appears to be almost the same but becomes brighter. However, upon further increasing the frequency to $7 \mathrm{kHz}$, the plasma appears diffused rather than having a chiral shape.

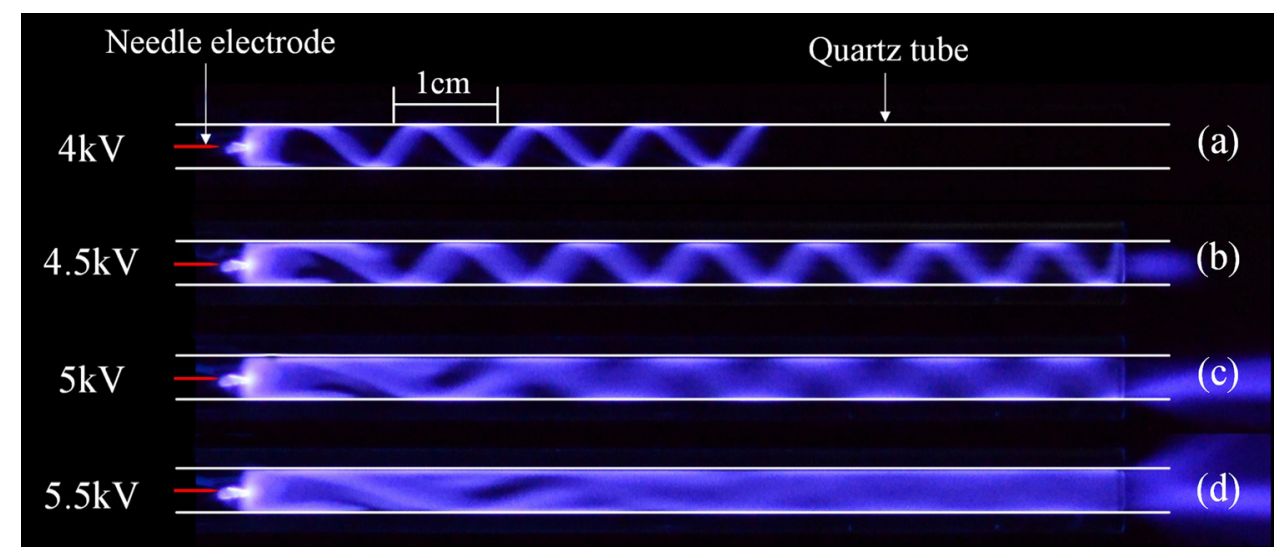

FIG. 3. Photographs of the plasma with different voltage amplitudes. (a)-(d) correspond to the voltage amplitudes of $4,4.5,5$, and $5.5 \mathrm{kV}$, respectively. The tube internal diameter is $4 \mathrm{~mm}$, the external diameter is $8 \mathrm{~mm}$, and the total length of the quartz tube is $10 \mathrm{~cm}$. Working gas: $\mathrm{N}_{2}$; gas pressure: $7 \mathrm{kPa}$, pulse frequency: $2 \mathrm{kHz}$, and pulse width: $1 \mu \mathrm{s}$. 


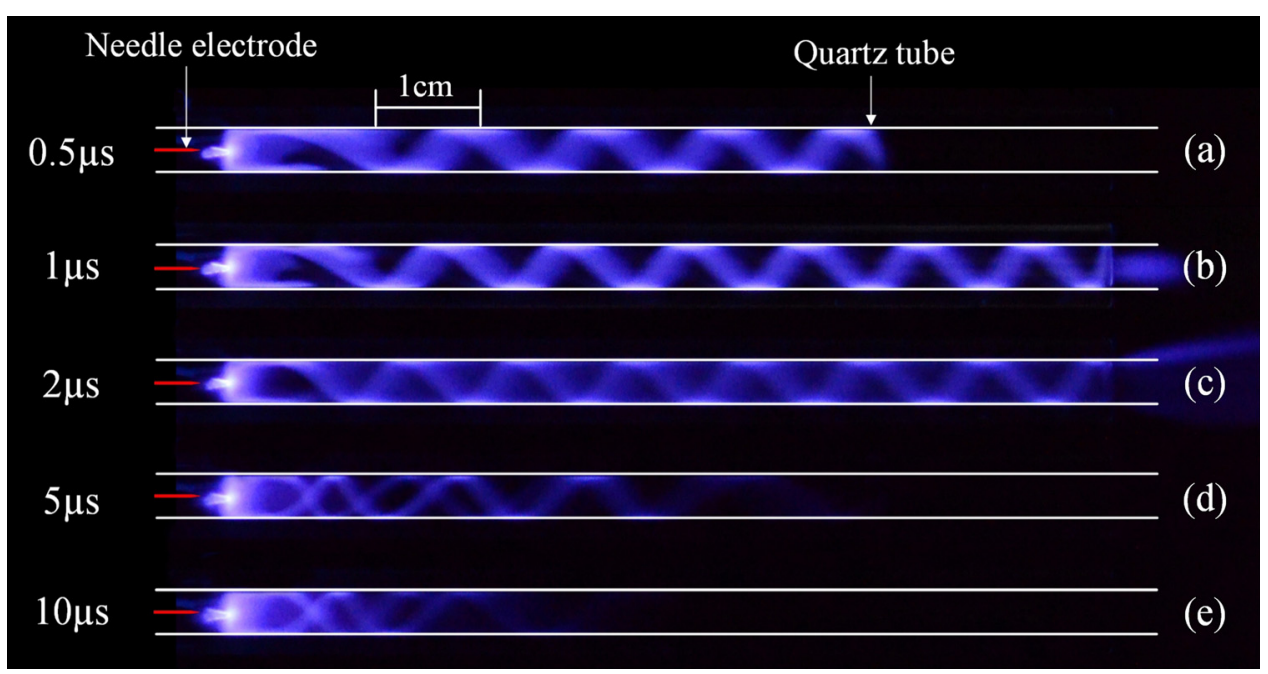

FIG. 4. Photographs of the plasma with different pulse widths of the applied voltage. (a)-(e) correspond to the pulse widths of $0.5,1,2,5$, and $10 \mu \mathrm{s}$, respectively. The tube internal diameter is $4 \mathrm{~mm}$, the external diameter is $8 \mathrm{~mm}$, and the total length of the quartz tube is $10 \mathrm{~cm}$. Working gas: $\mathrm{N}_{2}$, gas pressure: $7 \mathrm{kPa}$, amplitude of voltage: $4.5 \mathrm{kV}$, and pulse frequency: $2 \mathrm{kHz}$

\section{B. The effect of the shape of the tube on the chiral plasma}

Since quartz tube plays an important role in the formation of the chiral plasma, the shape of the quartz tube should affect the appearance of the plasma. Next, various shapes of tubes are studied to see how they affect the formation of the chiral plasma.

\section{Different thicknesses of the tube wall}

As discussed before, the chiral shaped plasma only appears in dielectric tube under certain limit conditions. It is suspected that the dielectric tube wall affects the impedance of the discharge and thus affects the appearance of the plasma. Therefore, the thickness of the tube wall probably affects the formation of the chiral plasma. Next, quartz tubes with four different thicknesses of the wall are used. As shown in Fig. 6(b), when the wall thickness is $2 \mathrm{~mm}$, a clear chiral plasma can be obtained. When the wall thickness is either reduced to $1 \mathrm{~mm}$ [Fig. 6(a)] or increased to $3 \mathrm{~mm}$ [Fig. 6(c)], only a vague chiral plasma can be observed. Upon further increasing the wall thickness to $4 \mathrm{~mm}$ as shown in Fig. 6(d), the plasma appears more or less diffused, and the chiral shape can hardly be seen.

Since electrical parameters also affect the formation of chiral shaped plasma, next, the pulse frequency is increased to $6 \mathrm{kHz}$ to see how it affects the appearance of the plasma for different wall thicknesses of the tube. As shown in Fig. 7, the chiral can be only obtained in the tube for the wall thickness of $2 \mathrm{~mm}$. A too thin or too thick wall is not favorable for forming the chiral shaped plasma.

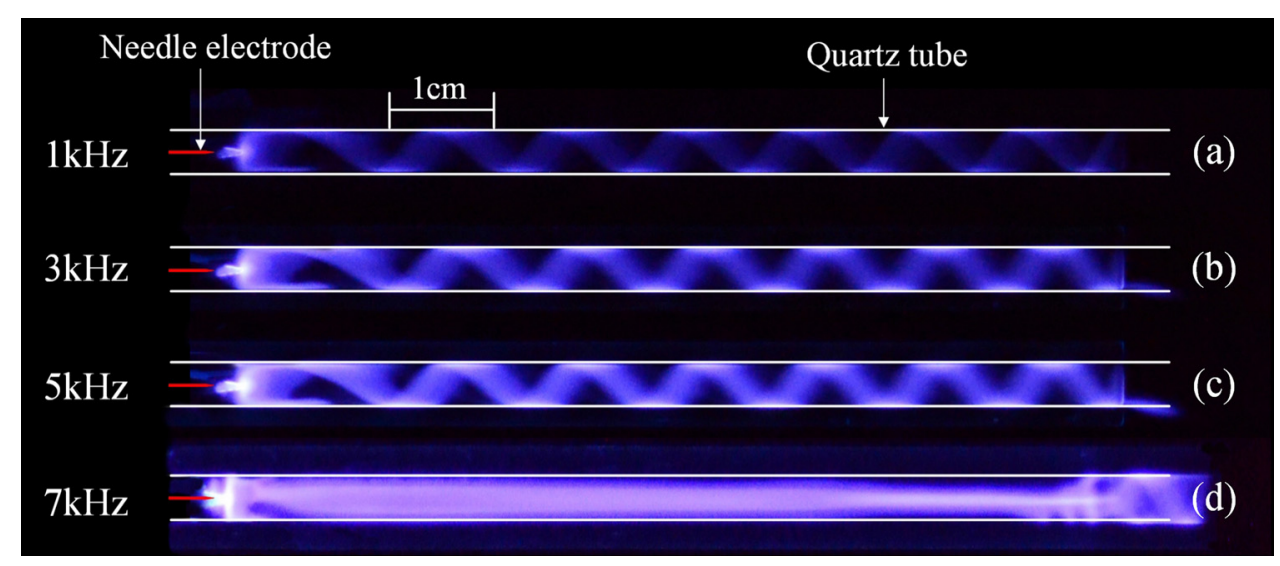

FIG. 5. Photographs of the plasma with different pulse frequencies of the applied voltage. (a)-(d) correspond to the pulse frequencies of $1,3,5$, and $7 \mathrm{kHz}$, respectively. The tube internal diameter is $4 \mathrm{~mm}$, the external diameter is $8 \mathrm{~mm}$, and the total length of the quartz tube is $10 \mathrm{~cm}$. Working gas: $N_{2}$, gas pressure: $7 \mathrm{kPa}$, amplitude of voltage: $4.5 \mathrm{kV}$, and pulse width: $1 \mu \mathrm{s}$. 


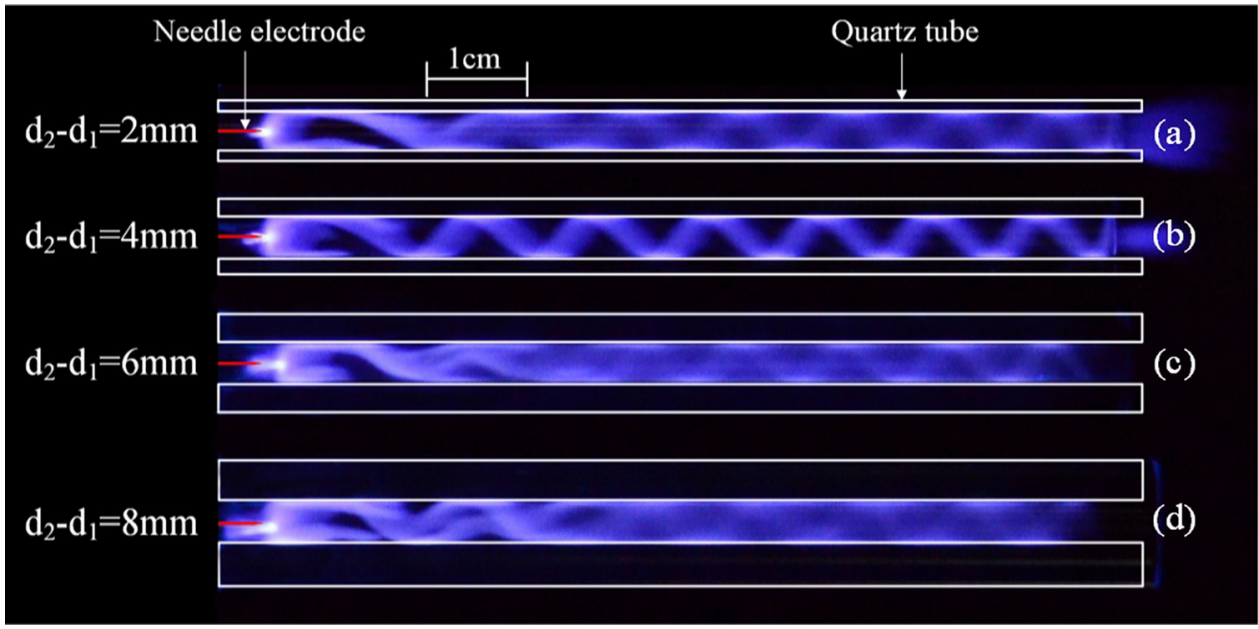

FIG. 6. Photographs of the plasma in quartz tubes with different thicknesses of the tube wall. (a)-(d) correspond to the thicknesses of $1,2,3$, and $4 \mathrm{~mm}$, respectively. Inner diameter $\mathrm{d}_{1}$ of all the tubes is $4 \mathrm{~mm}$, and the total length of all the tubes is $10 \mathrm{~cm}$. Working gas: $\mathrm{N}_{2}$; gas pressure: $7 \mathrm{kPa}$, amplitude of voltage: $4.5 \mathrm{kV}$, pulse frequency: $2 \mathrm{kHz}$, and pulse width: $1 \mu \mathrm{s}$.

\section{Abrupt changing the diameter of the tube}

First, the tube as shown in Fig. 1(d) is used, and the photographs of the plasma are shown in Fig. 8. Figure 8(b) shows that, when $d_{1}$ $=2 \mathrm{~mm}$, it is too small for the plasma to form a chiral shape. However, on the right side of the tube, when the inner diameter of the tube is increased to $4 \mathrm{~mm}$, a chiral plasma can be formed starting at the position of about $2 \mathrm{~cm}$ away from the joint position. Besides, it is found that upon either increasing or decreasing the gas pressure or increasing or reducing the applied voltage, the discharge cannot form the chiral shape in the tube with an inner diameter of $2 \mathrm{~mm}$. Figure 8 (c) shows that, when the inner diameter of the tube is reduced from $4 \mathrm{~mm}$ to $2 \mathrm{~mm}$, the chiral plasma formation in the left side of the tube cannot propagate into the right side of the tube. On the other hand, when we keep reducing the gas pressure, the plasma can gradually propagate into the right side of the tube, but it is diffused rather than having a chiral shape.

\section{Horn shape of the tube}

Since the diameter of the tube affects the formation of chiral plasma, next, the quartz tube with a horn shaped opening as shown in Fig. 1(c) is used, and the photographs of the plasma for different opening angles $\alpha$ are shown in Fig. 9. When $\alpha$ is $5^{\circ}$ as shown in Fig. 9(b), the plasma can still form a chiral shape with a much longer pitch. When $\alpha$ is increased to $10^{\circ}$ as shown in Fig. 9(c), a blurry chiral plasma can be seen, but it only has one turn. Upon increasing $\alpha$ to $20^{\circ}$, the chiral shaped plasma disappear, and the plasma only propagates

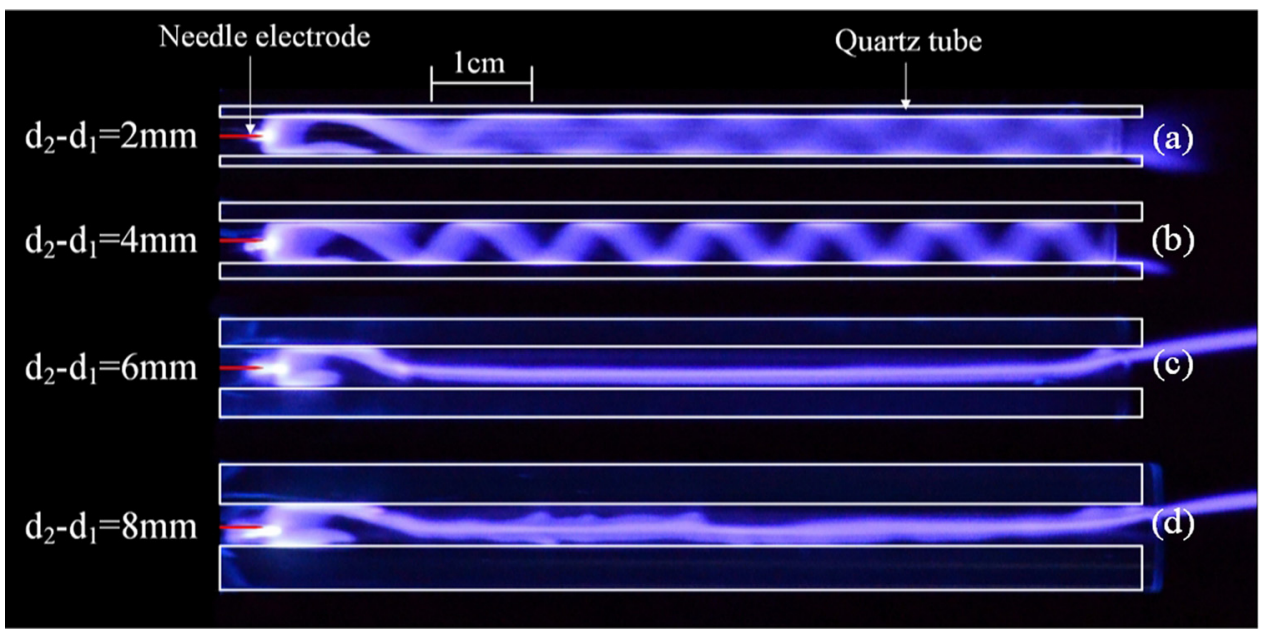

FIG. 7. Photographs of the plasma in quartz tubes with different thicknesses of the tube wall. (a)-(d) correspond to the thicknesses of $1,2,3$, and $4 \mathrm{~mm}$, respectively. Inner diameter $\mathrm{d}_{1}$ of all the tubes is $4 \mathrm{~mm}$, and the total length of all the tubes is $10 \mathrm{~cm}$. Working gas: $\mathrm{N}_{2}$; gas pressure: $7 \mathrm{kPa}$, amplitude of voltage: $4.5 \mathrm{kV}$, pulse frequency: $6 \mathrm{kHz}$, and pulse width: $1 \mu \mathrm{s}$. 


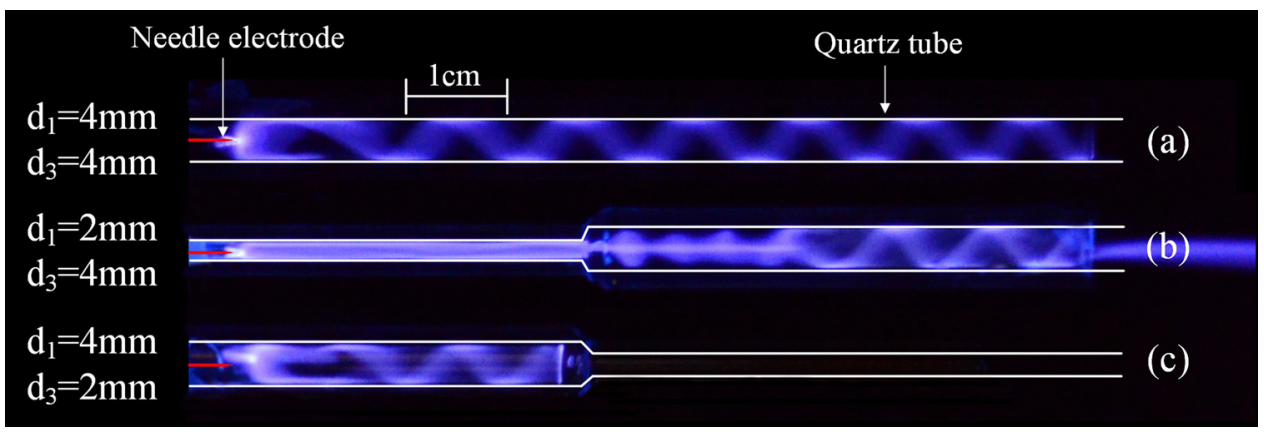

FIG. 8. Photographs of the plasma in quartz tubes with two different inner diameters connected. (a) the same inner diameter and (b) the inner diameters of the left side $d_{1}$ and right side $d_{3}$ of the tube are 2 and $4 \mathrm{~mm}$, respectively. (c) The inner diameters of the left side $d_{1}$ and right side $d_{3}$ of the tube are 4 and $2 \mathrm{~mm}$, respectively. The wall thickness of the tube is $2 \mathrm{~mm}$, and the total length of the tube is $10 \mathrm{~cm}$. Working gas: $\mathrm{N}_{2}$; gas pressure: $7 \mathrm{kPa}$, amplitude of voltage: $4.5 \mathrm{kV}$, pulse frequency: $2 \mathrm{kHz}$, and pulse width: $1 \mu \mathrm{s}$.

along one side of the horn tube. For the three horn shaped tubes with the different opening angles, when the applied voltage is reduced to $4 \mathrm{kV}$, the plasma only reaches the joint of the horn tube. On the other hand, when the applied voltage is increased to $5 \mathrm{kV}$, the plasma only propagates along one side of the horn tube, and no chiral shaped plasma can be observed.

As can be seen from Fig. 9, the chiral shaped plasma first forms in the straight part of the tube, and then it propagates into the horn shaped tube. Thus, the length of the straight part of the tube might affect the appearance of the plasma in the horn shaped tube. To see if this is true or not and to see how it affects the plasma behavior in the

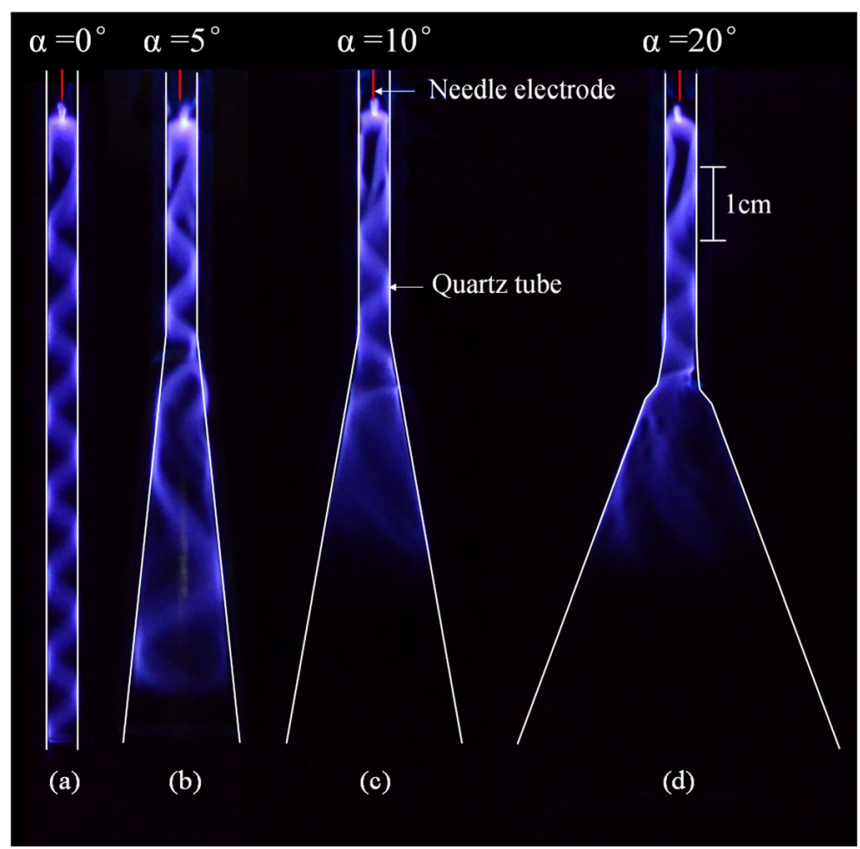

FIG. 9. Photographs of the plasma in quartz tubes of a horn shape with different opening angles. (a)-(d) correspond to the opening angles of $0,5,10$, and $20^{\circ}$, respectively. The total length of the tube is $10 \mathrm{~cm}$. The straight part of the tube for (b), (c), and (d) is $5 \mathrm{~cm}$. Working gas: $\mathrm{N}_{2}$; gas pressure: $7 \mathrm{kPa}$, amplitude of voltage: $4.5 \mathrm{kV}$, pulse frequency: $2 \mathrm{kHz}$, and pulse width: $1 \mu \mathrm{s}$. horn shaped tube, the different lengths $\mathrm{x}$ of the straight part of the tube with the same opening angle $\alpha$ of $5^{\circ}$ are studied. As shown in Fig. 10 , with the decrease in $\mathrm{x}$, the turns of the chiral shaped plasma in the horn shaped tube gradually increases.

\section{Square quartz tube}

For all the experiments discussed above, only circular tubes are used. If square tube is used, it would disrupt the axial symmetry of the system and may result in the disappearance of the chiral shape of the plasma. To see if this is true or not, a square quartz tube is used in the

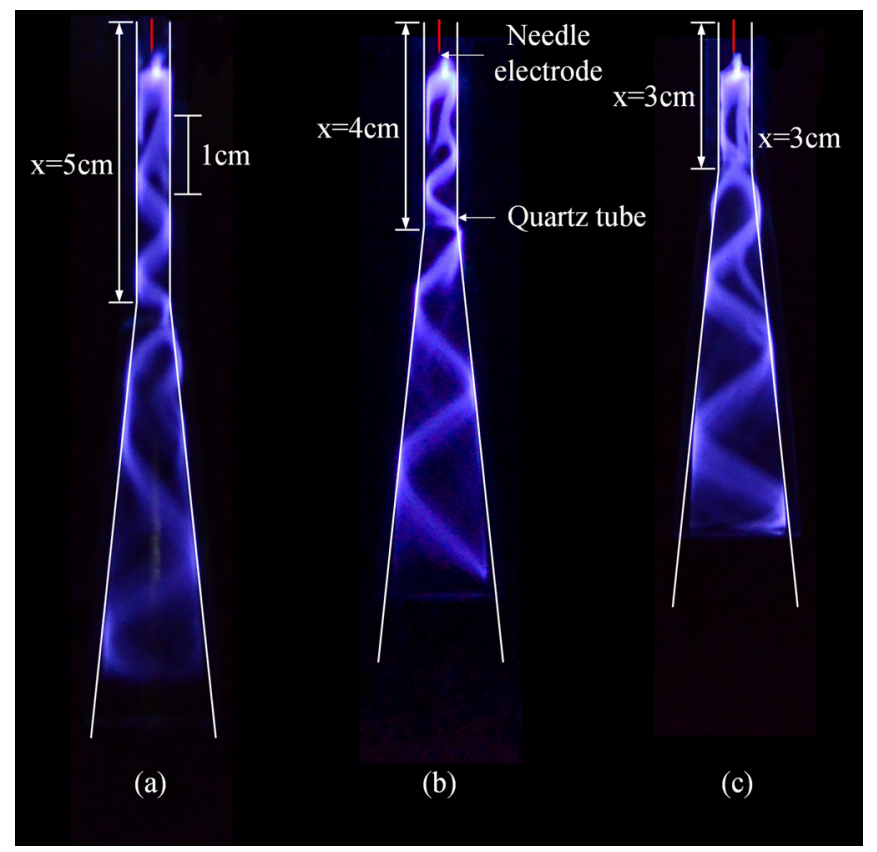

FIG. 10. Photographs of the plasma in quartz tube of horn shape with different lengths of the straight part. The opening angle is $5^{\circ}$ for all the three cases. (a)-(c) correspond to the length of the straight part of 5,4 , and $3 \mathrm{~cm}$, respectively. Working gas: $\mathrm{N}_{2}$; gas pressure: $7 \mathrm{kPa}$, amplitude of voltage: $4.5 \mathrm{kV}$, pulse frequency: $2 \mathrm{kHz}$, and pulse width: $1 \mu \mathrm{s}$. 


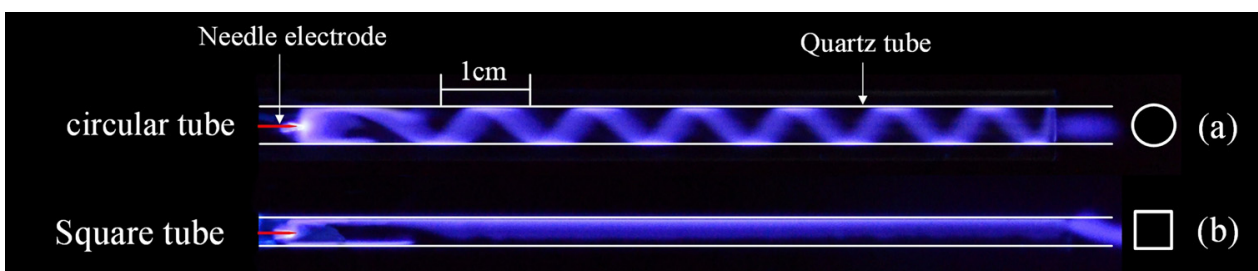

FIG. 11. Photographs of the plasma in circular and square quartz tubes. (a) Circular quartz tube with an inner diameter of $4 \mathrm{~mm}$ and (b) square quartz tube with the length of its sides of $4 \mathrm{~mm}$.

following experiments. The plasma in the square quartz tube is shown in Fig. 11(b). Different from the plasma in circular quartz tube [Fig. 11(a)], the plasma in the square quartz tube only propagates along one side.

Finally, to see if an already formed chiral plasma can propagate in a square tube, circular tubes with different lengths are connected to square tube as shown in Fig. 1(f). The plasmas are shown in Fig. 12. It can be seen that there is still no chiral shaped plasma in the square tube, and even the chiral plasmas already formed in the left side of the circular tubes.

\section{The effect of the electrode position on the chiral plasma}

For all the experimental results discussed above, the high voltage electrode is placed at the center of the tube. If the electrode is placed away from the center of the tube, the symmetrical characteristic of the discharge will be disrupted, which might affect the appearance of the chiral plasma. Next, the high voltage electrode is placed at different distances $s$ from the top inner wall of the quartz tube. $s=0$ means that it is placed next to the top inner wall of the quartz tube. $s=2,3$, and $4 \mathrm{~mm}$ correspond to the center of the tube, $1 \mathrm{~mm}$ lower away from the center of the tube, and at the bottom inner wall of the tube, respectively. Figure 13 shows the photographs of the plasma for $s=0,2,3$, and $4 \mathrm{~mm}$, respectively. It shows that the change of $\mathrm{s}$ does not affect the formation of the chiral plasma plume. Either the pitch or the total length of the chiral plasma is the same for all the different $s$ values.

\section{DISCUSSION AND CONCLUSION}

In previous works, the effects of gas pressure, applied voltage, and other parameters on the chiral plasma were investigated. The additional helical coil and ring were applied to investigate how they would affect the appearance of the plasma. It is found that the formation of the chiral plasma plume occurs only under limited experimental conditions. It is noted that all previous experiments used only circular tube, and the tube plays an important role in the formation of the chiral plasma plume. In this paper, to have better understanding of how the chiral plasma is generated, in addition to the effect of the electric parameters on the chiral plasma, the effects of the tube geometry on the chiral plasma are investigated.

First, the thickness of dielectric tube would affect the impedance of the discharge circuit; thus, it might affect the appearance of the plasma plume. It is interesting to find that a too thin or too thick tube wall is not favorable for generating the chiral plasma. The chiral plasma plume can be obtained only at the tube wall thickness of $2 \mathrm{~mm}$. It is interesting to point out that, when the experiment was designed, it was assumed that a thicker tube wall could result in larger capacitance of the plasma to the virtual ambient ground, and thus, the

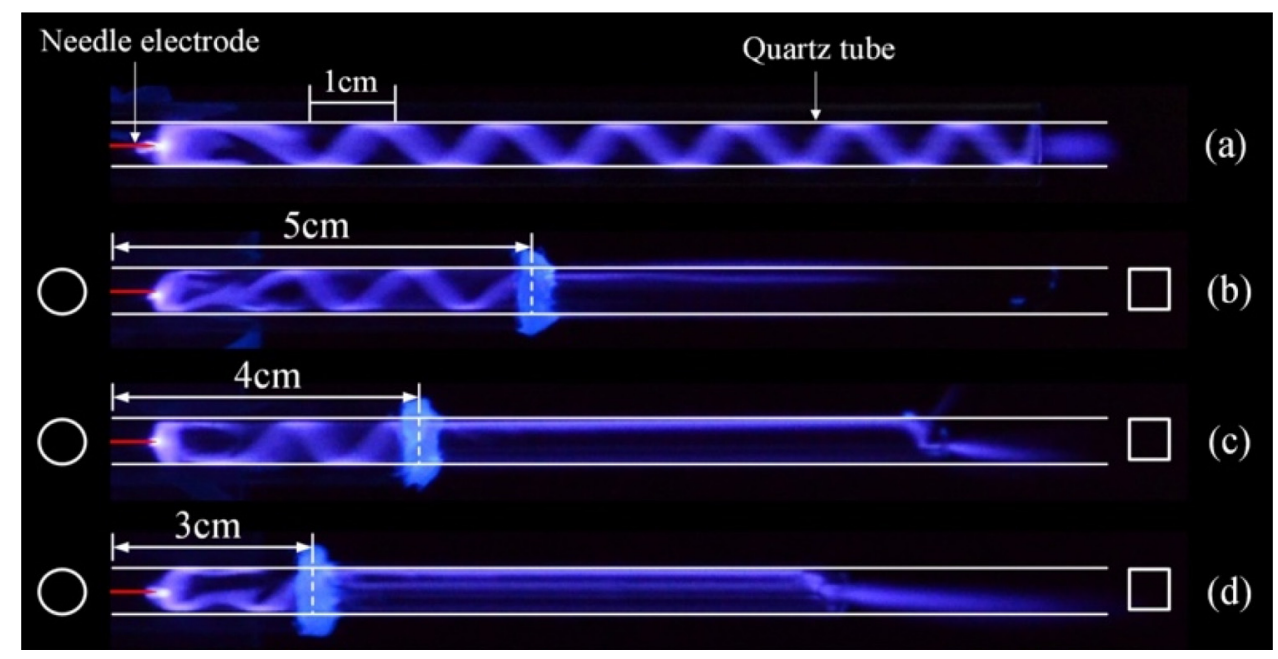

FIG. 12. Photographs of the plasma in quartz tube where the left part is made of circular tube and the right part is made of square tube. (a) Circular quartz tube; for (b)-(d), the left part of the circular quartz tube is $5 \mathrm{~cm}, 4 \mathrm{~cm}$, and $3 \mathrm{~cm}$, respectively. 


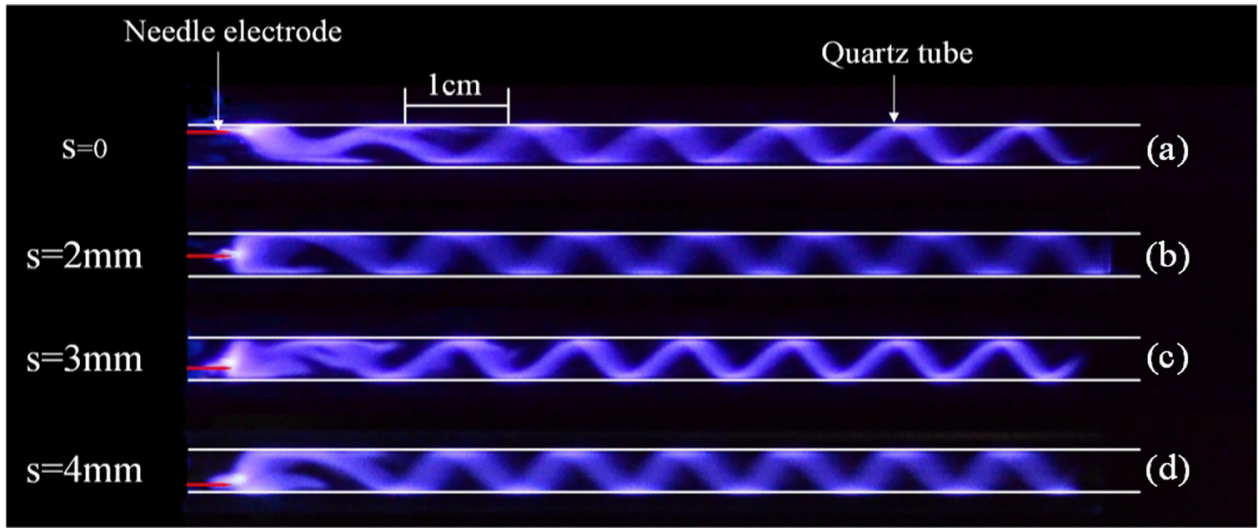

FIG. 13. Photographs of the plasma with different positions of the high voltage electrode. $s$ is the distance between the electrode and the up inner quartz tube wall. (a)-(d) correspond to the distance between the electrode and the quartz tube wall being $0,2,3$, and $4 \mathrm{~mm}$, respectively. The tube internal diameter is $4 \mathrm{~mm}$, the external diameter is $8 \mathrm{~mm}$, and the length is $10 \mathrm{~cm}$. Working gas: $\mathrm{N}_{2}$; gas pressure: $7 \mathrm{kPa}$, amplitude of voltage: $4.5 \mathrm{kV}$, pulse frequency: $2 \mathrm{kHz}$, and pulse width: $1 \mu \mathrm{s}$.

electric field component on the radial direction would be increased, which might result in a shorter pitch of the plasma plume. Certainly, this was not what was observed, and further investigation is needed.

Second, the effect of the diameter of the tube on the chiral plasma is investigated. It is found that, when a tube with an inner diameter of $2 \mathrm{~mm}$ is connected with a tube with an inner diameter of $4 \mathrm{~mm}$ and the high voltage electrode is placed in the tube with an inner diameter of $2 \mathrm{~mm}$, a diffuse plasma is generated in the tube with an inner diameter of $2 \mathrm{~mm}$, it propagates into the tube with an inner diameter of $4 \mathrm{~mm}$, and a chiral plasma can be obtained in the larger diameter tube. On the other hand, when the high voltage electrode is placed in the tube with an inner diameter of $4 \mathrm{~mm}$, the chiral plasma formation in the larger diameter tube cannot propagate into the small diameter tube.

Third, horn shaped tubes with different opening angles are tested. When the opening angle is $5^{\circ}$, the plasma can still form a chiral shape with a much longer pitch. This might be explained as follows: for the horn shaped tubes, with the propagation of the plasma plume, it is equivalent to the increase in the inner diameter of the tube and the ratio between the electric field components on the axial direction and on the radial direction might be increased, which might be the reason for the longer pitch.

When the opening angle is increased to $10^{\circ}$, a blurry chiral plasma can still be seen, but it only has one turn. When we keep increasing the opening angle to $20^{\circ}$, the chiral shaped plasma disappears, and the plasma only propagates along one side of the horn tube.

Fourth, to disrupt the axial symmetry, square quartz tubes are used. It is found that the plasma in the square quartz tube only propagates along one side of the tube wall.

Finally, the high voltage electrode is placed away from the center of the circular tube to disrupt its symmetry, and it is found that the chiral plasma plume can always be seen even if the high voltage is placed next to the inner wall of the tube. Either the pitch or the total length of the chiral plasma is the same for all the different $s$ values. This phenomenon is unexpected. According to the observed chiral plasma plume, it keeps the same symmetry when the high voltage needle electrode is shifted from the center of the tube. Thus, we can conclude that the magnetic field induced by the current flow on the needle electrode does not play the essential role in the propagation of the chiral plasma plume. This is because, when the needle electrode is shifted from the center of the tube, the magnetic field induced by the current flow on the needle would not be symmetric along the tube, and the chiral plasma plume should lose symmetry. But this was not observed, which might indicate that the magnetic field induced by the current flow along the needle electrode plays a minor role in the formation of the chiral plasma plume.

In short, in this paper, by using different shapes of the tubes and by shifting the electrode away from the center to disrupt the symmetry, we have better understanding of the chiral plasma plume behavior. We know more about the conditions to generate the chiral plasma, such as the suitable thickness and diameter of the tube for obtaining clear chiral plasma; a too large opening angle of the horn tube is not favorable for the propagation of the chiral plasma. However, there are still many questions which need to be answered. For example, for different thicknesses of tubes, only when the thickness is $2 \mathrm{~mm}$, a clear chiral plasma can be observed; the reason is not clearly understood. We thought that the thicker wall of the tube would result in lower impedance of the discharge circuit, and thus, it might be favorable for generating the chiral plasma. But experimental results show that this is obviously not the case. Nevertheless, why the plasma assumes a chiral shape is not understood yet. More experiments including simulations are needed to understand the chiral plasma behavior.

\section{ACKNOWLEDGMENTS}

We would like to thank Professor Mark Kushner and Professor Hae June Lee for great discussions and suggestions. This work was supported by the National Natural Science Foundation of China (Grant Nos. 51625701 and 51977096).

\section{REFERENCES}

${ }^{1}$ A. Shashurin, M. N. Shneider, A. Dogariu, R. B. Miles, and M. Keidar, Appl. Phys. Lett. 94, 231504 (2009).

${ }^{2}$ S. Park, U. Cvelbar, W. Choe, and S. Y. Moon, Nat. Commun. 9, 371 (2018).

${ }^{3}$ N. Yu. Babaeva, G. V. Naidis, and M. J. Kushner, Plasma Sources Sci. Technol, 27, 015016 (2018).

${ }^{4}$ F. Tholin and A. Bourdon, Plasma Phys. Controlled Fusion 57, 014016 (2015). 
${ }^{5}$ G. V. Naidis, Plasma Processes Polym. 14, 1600127 (2017).

${ }^{6}$ C. Douat, G. Bauville, M. Fleury, M. Laroussi, and V. Puech, Plasma Sources Sci. Technol. 21, 034010 (2012).

7J. L. Walsh, F. Iza, N. B. Janson, V. J. Law, and M. G. Kong, J. Phys. D: Appl. Phys. 43, 075201 (2010).

${ }^{8}$ W. van Gaens and A. Bogaerts, Plasma Sources Sci. Technol. 23, 035015 (2014).

${ }^{9}$ E. Robert, V. Sarron, T. Darny, D. Riès, S. Dozias, J. Fontane, L. Joly, and J.-M. Pouvesle, Plasma Sources Sci. Technol. 23, 012003 (2014).

${ }^{10}$ M. Janda, Z. Machala, A. Niklová, and V. Martišovitš, Plasma Sources Sci. Technol. 21, 045006 (2012).

${ }^{11}$ S. Kos, T. Blagus, M. Cemazar, G. Filipic, G. Sersa, and U. Cvelbar, PLoS One 12, e0174966 (2017).

${ }^{12}$ T. He, D. Liu, Z. Liu, Z. Liu, Q. Li, M. Rong, and M. Kong, Appl. Phys. Lett. 111, 203702 (2017).

${ }^{13}$ E. J. Szili, J. Bradley, and R. Short, J. Phys. D: Appl. Phys. 47, 152002 (2014).

${ }^{14} \mathrm{~S}$. Wu, X. Lu, D. Liu, Y. Yang, Y. Pan, and K. Ostrikov, Phys. Plasmas 21, 103508 (2014).

${ }^{15}$ X. Pei, D. Gidon, Y. Yang, Z. Xiong, and D. Graves, Chem. Eng. J. 362, 217 (2019).

${ }^{16}$ X. Lu, M. Keidar, M. Laroussi, E. Choi, E. Szili, and K. Ostrikov, Mater. Sci. Eng. R 138, 36 (2019).

${ }^{17}$ M. Teschke, J. Kedzierski, E. G. Finantu-Dinu, D. Korzec, and J. Engemann, IEEE Trans. Plasma Sci. 33, 310 (2005).
${ }^{18} \mathrm{X}$. Lu and M. Laroussi, J. Appl. Phys. 100, 063302 (2006).

${ }^{19}$ J. Boeuf, L. Yang, and L. Pitchford, J. Phys. D: Appl. Phys. 46, 015201 (2013).

${ }^{20}$ G. V. Naidis, J. Phys. D: Appl. Phys. 43, 402001 (2010).

${ }^{21}$ M. Yousfi, O. Eichwald, N. Merbahi, and N. Jomma, Plasma Sources Sci. Technol. 21, 045003 (2012).

${ }^{22}$ Z. Xiong and M. J. Kushner, Plasma Sources Sci. Technol. 21, 034001 (2012).

${ }^{23}$ X. Lu and K. Ostrikov, Appl. Phys. Rev. 5, 031102 (2018).

${ }^{24}$ Y. Xian, D. Zou, X. Lu, Y. Pan, and K. Ostrikov, Appl. Phys. Lett. 103, 094103 (2013).

${ }^{25}$ D. Zou, X. Cao, X. Lu, and K. Ostrikov, Phys. Plasmas 22, 103517 (2015).

${ }^{26}$ T. Darny, E. Robert, D. Riès, S. Dozias, and J. Pouvesle, IEEE Trans. Plasma Sci. 42, 2504 (2014).

${ }^{27}$ T. Darny, E. Robert, S. Dozias, and J. Pouvesle, IEEE Trans. Plasma Sci. 42, 2506 (2014).

${ }^{28}$ J. Jánský, P. Delliou, F. Tholin, P. Tardiveau, A. Bourdon, and S. Pasquiers, J. Phys. D: Appl. Phys. 44, 335201 (2011).

${ }^{29}$ A. Meshchanov, A. Korshunov, Y. Ionikh, and N. Dyatko, Plasma Phys. Rep. 41, 677 (2015).

${ }^{30}$ Y. Akishev, G. Aponin, A. Balakirev, M. Grushin, V. Karalnik, A. Petryakov, and N. Trushkin, Plasma Sources Sci. Technol. 20, 024005 (2011).

${ }^{31}$ L. Nie, F. Liu, X. Zhou, X. Lu, and Y. Xian, Phys. Plasmas 25, 053507 (2018).

${ }^{32}$ F. Liu, J. Li, F. Wu, L. Nie, and X. Lu, J. Phys. D: Appl. Phys. 51, 294003 (2018). 